KEK-TH-770

May, 2001

\title{
Dynamical Doublet-Triplet Higgs Mass Splitting
}

\author{
Ryuichiro Kitano ${ }^{a b *}$ and Nobuchika Okada ${ }^{a \dagger}$ \\ ${ }^{a}$ Theory Group, KEK, Oho 1-1, Tsukuba, Ibaraki 305-0801, Japan \\ ${ }^{b}$ Department of Particle and Nuclear Physics, The Graduate University for Advanced Studies, \\ Oho 1-1, Tsukuba, Ibaraki 305-0801, Japan
}

\begin{abstract}
We propose a new mechanism toward the solution to the doublet-triplet Higgs mass splitting problem in the supersymmetric grand unified theory. Our model is based on the gauge group $S U(5)_{H} \times S U(5)_{G U T}$, where $S U(5)_{H}$ and $S U(5)_{G U T}$ are a new strong gauge interaction and the ordinary grand unified gauge group, respectively. The doublet-triplet Higgs mass splitting is realized through the quantum deformation of moduli space caused by the strong $S U(5)_{H}$ gauge dynamics. The low energy description of our model is given by the minimal supersymmetric standard model.
\end{abstract}

\footnotetext{
*e-mail: ryuichiro.kitano@kek.jp

†e-mail: okadan@camry.kek.jp
} 


\section{INTRODUCTION}

Supersymmetric extension is one of the the most promising ways in order to provide a solution to the gauge hierarchy problem in the standard model [1]. The minimal version of this extension of the standard model is called the minimal supersymmetric standard model (MSSM). Interestingly, the experimental data support the unification of three gauge couplings at the scale $M_{G U T} \sim 10^{16} \mathrm{GeV}$ with only the MSSM particle contents [2]. This fact seems to strongly suggest the original idea of the grand unified theory (GUT) [3] together with supersymmetry (SUSY), that is, SUSY GUT. At high energy, the theory may be described by a simple gauge group such as $S U(5)$ into which the standard model is embedded.

However, the GUT suffers from a serious problem, namely, the doublet-triplet Higgs mass splitting problem. While the doublet parts of the Higgs superfields in $\overline{\mathbf{5}}$ and $\mathbf{5}$ representations (in terminology of $S U(5)$ GUT) should have the electroweak scale mass, the triplet part Higgs masses have to be in the grand unification scale in order to avoid rapid proton decay [4] and not to destroy the successful gauge coupling unification. In a simple treatment, a parameter in the theory should be chosen with accuracy of $10^{-14}$ to realize this mass splitting. Although, in a supersymmetric theory, this fine-tuning is "technically natural" because of the non-renormalization theorems [5], this is basically the same problem as the original gauge hierarchy problem. Thus, the theory may not be compelling as a resolution of the hierarchy problem, unless this mass splitting is naturally realized without the fine-tuning.

There have been many efforts to solve the doublet-triplet Higgs mass splitting problem. These are roughly classified into two approaches. One is the dynamical approach such as the sliding singlet mechanism [6]. The other is the group theoretical one such as, for example, the missing partner mechanism [7] [8], the Dimopoulos-Wilczek mechanism [9], the idea of Higgs doublets as pseudo Nambu-Goldstone bosons [10. More recently, an alternative approach was investigated based on the extra-dimensional theories [11.

In this paper, we propose a new mechanism which realizes the doublet-triplet Higgs mass splitting through the strong gauge dynamics. Our model is based on the gauge group $S U(5)_{H} \times S U(5)_{G U T}$, where $S U(5)_{H}$ is the strong gauge interaction additionally introduced to the ordinary GUT gauge group $S U(5)_{G U T}$. At low energy, the $S U(5)_{H}$ gauge interaction becomes strong, and the theory is described by the effective field $M$ of the $\mathbf{2 5}(=\mathbf{1}+\mathbf{2 4})$ representation under the $S U(5)_{G U T}$ gauge group. In our model, this effective field has the effective Yukawa coupling among the pair of Higgs superfields $(\bar{H}$ and $H)$ such as $W=$ $\bar{H} M H$. This is the unique source for the Higgs masses. The strong $S U(5)_{H}$ dynamics causes the quantum deformation of moduli space, whose SUSY vacuum condition requires that the effective field should have, at least, two zero eigenvalues. On the desired vacuum where the GUT gauge symmetry is correctly broken to the MSSM gauge group, this means that the strong dynamics requires that the pair of Higgs doublets are massless, but the pair of Higgs triplets are heavy through the effective Yukawa coupling. Therefore, the doublettriplet Higgs mass splitting is realized through the effect of the strong gauge dynamics. This is a new mechanism, we propose, to solve the doublet-triplet Higgs mass splitting problem.

In the next section, we discuss the dynamical sector of our model and the realization of the doublet-triplet Higgs mass splitting through the strong gauge dynamics. Our model has some non-renormalizable superpotentials which are necessary for our mechanism to work. The origins of them are discussed in Sec. III. Also, a concrete model as an example 
is presented, in which the $S U(5)_{G U T}$ gauge symmetry is correctly broken to the standard model gauge group and the light particle contents are the same as that of the MSSM. In Sec. IV, we summarize our discussion and give some comments.

\section{DYNAMICAL DOUBLET-TRIPLET HIGGS MASS SPLITTING}

In this section, we discuss the dynamical sector of our model in which the doublet-triplet Higgs mass splitting is realized through the strong gauge dynamics. This sector is based on the gauge group $S U(5)_{H} \times S U(5)_{G U T}$ with the particle contents as follows.

$\begin{array}{cccc} & S U(5)_{H} & S U(5)_{G U T} & U(1)_{R} \\ \bar{Q} & \overline{\mathbf{5}} & \overline{\mathbf{5}} & 2 / 5 \\ Q & \mathbf{5} & \mathbf{5} & 2 / 5 \\ \bar{H} & \mathbf{1} & \overline{\mathbf{5}} & 4 / 5 \\ H & \mathbf{1} & \mathbf{5} & 2 / 5\end{array}$

Here, $\bar{H}$ and $H$ are the ordinary Higgs superfields in the $S U(5)_{G U T}$ theory, and $U(1)_{R}$ is the R-symmetry. I Note that any renormalizable superpotentials are forbidden by the Rsymmetry. In the following, we consider some non-renormalizable superpotentials consistent with all the symmetries. Their origins are discussed in Sec. III based on all the renormalizable superpotentials.

Let us first consider the superpotential of the form

$$
W=\bar{b} \bar{Q}^{5}+b Q^{5}
$$

where $\bar{Q}^{5}$ and $Q^{5}$ denote the contraction of the gauge indices by the epsilon tensors, and $\bar{b}$ and $b$ are the constants with the mass dimension -2 . At low energy, the $S U(5)_{H}$ gauge interaction becomes strong. We can describe the low energy effective theory by the method of Seiberg and co-workers [12]. The moduli space is deformed by the strong gauge dynamics so as to satisfy the condition, $\operatorname{det} M / \Lambda^{3}-\bar{B} B=\Lambda^{2}$, where $\Lambda$ is the dynamical scale of the gauge interaction whose scale is assumed as $\Lambda \sim M_{G U T}$, and $M, \bar{B}$ and $B$ are the mesonic and baryonic effective fields, which are singlet under $S U(5)_{H}$, as follows.

$\begin{array}{rccc} & & S U(5)_{G U T} & U(1)_{R} \\ M & \sim(\bar{Q} Q) / \Lambda & \mathbf{1 + 2 4} & 4 / 5 \\ \bar{B} & \sim \bar{Q}^{5} / \Lambda^{4} & \mathbf{1} & 2 \\ B & \sim Q^{5} / \Lambda^{4} & \mathbf{1} & 2\end{array}$

The dynamically generated constraint is incorporated in the superpotential by introducing the Lagrange multiplier superfield $X$, and the total effective superpotential is given by

\footnotetext{
1 This R-symmetry is anomalous with respect to the $S U(5)_{H}$ gauge interaction, and broken down to the discrete symmetry $Z_{5}$ (see Eq. (2)). It is possible to introduce the discrete $Z_{5}$ symmetry from the beginning, instead of the $U(1)_{R}$ symmetry. In any case, we obtain the same results.
} 


$$
W=\Lambda^{4} \bar{b} \bar{B}+\Lambda^{4} b B+X\left(\frac{\operatorname{det} M}{\Lambda^{3}}-\bar{B} B-\Lambda^{2}\right) .
$$

Considering the SUSY vacuum conditions for the heavy fields, $\bar{B}, B$ and $X$, and integrating them out, we obtain a more convenient form of the effective superpotential such that

$$
W= \pm \Lambda^{4}(\bar{b} b)^{1 / 2}\left(\frac{\operatorname{det} M}{\Lambda^{3}}-\Lambda^{2}\right)^{1 / 2}
$$

The SUSY vacuum condition for $M$ leads to

$$
(\operatorname{det} M) M^{-1}=0,
$$

which means that the effective field $M$ should have, at least, two zero eigenvalues, namely, $M=\operatorname{diag}\left(a_{1}, a_{2}, a_{3}, 0,0\right)$. Although, in general, $a_{i}(i=1,2,3)$ are arbitrary constants, they are assumed to be $a_{1}=a_{2}=a_{3}=v \sim M_{G U T}$ in order to obtain the correct $S U(5)_{G U T}$ symmetry breaking pattern, $S U(5)_{G U T} \rightarrow S U(3)_{c} \times S U(2)_{L} \times U(1)_{Y}$. We will present a model to realize this situation in the next section.

Now, we show that the doublet-triplet Higgs mass splitting is realized through the effects of the strong gauge dynamics. Suppose the tree level superpotential such as f

$$
W=\lambda H^{i}\left(\bar{Q}_{\alpha i} Q^{\alpha j}\right) \bar{H}_{j},
$$

where $\alpha$ and $i, j$ are the gauge indices of $S U(5)_{H}$ and $S U(5)_{G U T}$, respectively, and $\lambda$ is a constant with the mass dimension -1 . At low energy, this superpotential is described in terms of the effective field $M$, and leads to the Yukawa coupling

$$
W=(\lambda \Lambda) \bar{H} M H .
$$

Note that, as discussed above, the strong gauge dynamics requires the eigenvalues for $M$ such that $M=\operatorname{diag}(v, v, v, 0,0)$. Therefore, the doublet-triplet Higgs mass splitting is realized through the effect of the strong gauge dynamics. This is the point of this paper.

The effective description of our model is similar to the models with the sliding singlet mechanism [6]. In these models, the same superpotential as Eq. (6) is introduced, and $M=\operatorname{diag}(v, v, v, 0,0)$ is realized through the SUSY vacuum conditions, $\partial W / \partial \bar{H}=0$ and $\partial W / \partial H=0$, providing the vacuum expectation value (VEV) for the doublet part of the Higgs fields. However, these models suffer from the tadpole problem [13] [14], once the SUSY breaking effects in supergravity scenario are taken into account. Note that the direction to the non-zero F-terms, $\partial W / \partial \bar{H}$ and $\partial W / \partial H$, is bounded by the VEV of the Higgs doublets (the electroweak scale), and is almost flat compared with the typical SUSY breaking scale. Thus, the vacuum destabilization occurs, once the tadpole terms of the singlet field are induced through the supergravity effects. As a result, the SUSY vacuum on which the

2 The absence of the term $\bar{H}_{i} H^{i}\left(\bar{Q}_{\alpha j} Q^{\alpha j}\right)$ is crucial for our mechanism to work. The reason why we do not introduce the term will be explained in the next section. 
doublet Higgs superfields are massless is smoothed out, and the sliding singlet mechanism cannot work. 3 On the other hand, note that, in our model, the massless-ness of the doublet Higgs superfields is required by the strong gauge dynamics whose scale is much larger than the typical SUSY breaking scale. Thus, the stability of the SUSY vacuum is rigidly ensured, and our model is free from the tadpole problem. This is the crucial difference of our mechanism from the sliding singlet mechanism.

\section{A CONCRETE MODEL}

Now we present a concrete model as an example, in which our mechanism discussed in the previous section can work and there is the desired SUSY vacuum.

Let us first discuss the origins of the non-renormalizable superpotentials in Eqs. (1) and (5). In the following discussion, we always consider the case that all the fundamental superpotentials are renormalizable. In this sense, the non-renormalizable terms are effective ones which are originated from integrating out heavy superfields according to the SUSY vacuum conditions. What types of non-renormalizable superpotentials are induced depends on the particle contents of the model. The heavy particle contents, in our model, are as follows.

$\begin{array}{cccc} & S U(5)_{H} & S U(5)_{G U T} & U(1)_{R} \\ \bar{\Phi}_{1} & \overline{\mathbf{1 0}} & \overline{\mathbf{1 0}} & 4 / 5 \\ \bar{\Phi}_{2} & \overline{\mathbf{1 0}} & \overline{\mathbf{1 0}} & 6 / 5 \\ \Phi_{1} & \mathbf{1 0} & \mathbf{1 0} & 6 / 5 \\ \Phi_{2} & \mathbf{1 0} & \mathbf{1 0} & 4 / 5 \\ \bar{A} & \overline{\mathbf{5}} & \mathbf{1} & 4 / 5 \\ A & \mathbf{5} & \mathbf{1} & 6 / 5\end{array}$

The general renormalizable superpotential including $\bar{\Phi}_{j}$ and $\Phi_{j}(j=1,2)$ is found to be

$$
\begin{aligned}
W & =\bar{Q} \bar{Q} \Phi_{1}+\bar{Q} \bar{\Phi}_{1} \bar{\Phi}_{1}+m_{1} \bar{\Phi}_{1} \Phi_{1} \\
& +Q Q \bar{\Phi}_{2}+Q \Phi_{2} \Phi_{2}+m_{2} \bar{\Phi}_{2} \Phi_{2}
\end{aligned}
$$

where the gauge indices are appropriately contracted, $m_{1}$ and $m_{2}$ are the mass terms, and we take all the Yukawa coupling constants to be 1 , for simplicity. Since $\bar{\Phi}_{j}$ and $\Phi_{j}$ are charged under the $S U(5)_{H}$ gauge group, they should be heavy enough, at least, heavier than the dynamical scale $\Lambda$ in order not to change the $S U(5)_{H}$ dynamics discussed in the previous section. After integrating the heavy superfields out, we obtain the effective superpotential in Eq. (1) with the relations $\bar{b}=1 / m_{1}^{2}$ and $b=1 / m_{2}^{2}$. f

3 There is a possibility to avoid this problem 15 by considering the low scale SUSY breaking scenario, such as the gauge mediated SUSY breaking scenario [16].

${ }^{4}$ Even if we take $m_{1} \sim m_{2} \sim M_{P}$ ( $M_{P}$ is the Planck mass), the coefficients of the tadpole terms for $\bar{B}$ and $B$ are much larger than the typical SUSY breaking scale. No destabilization occurs for the SUSY vacuum we discussed, even if the SUSY breaking effects are taken into account. 
The effective superpotential in Eq. (5) is induced as follows. The general renormalizable superpotential including $\bar{A}$ and $A$ is given by

$$
W=\bar{A} Q \bar{H}+A \bar{Q} H+m_{A} \bar{A} A,
$$

where $m_{A}$ is the mass parameter, and we take all the Yukawa couplings to be 1, for simplicity. This mass term should be large enough, at least, larger than $\Lambda$. We assume that $m_{A}$ is slightly larger than $\Lambda$. Integrating out $\bar{A}$ and $A$ leads to Eq. (5) with the relation $\lambda=$ $-1 / m_{A} \sim-1 / \Lambda$. Note that the term $\bar{H}_{i} H^{i}\left(\bar{Q}_{\alpha j} Q^{\alpha j}\right)$ which destroys our mechanism is not generated with the above particle contents. This is the reason why we introduced only the superpotential of Eq. (5) in the previous section.

Finally, let us consider the superpotential including the effective field $M$. Through this superpotential, we can obtain the desired SUSY vacuum where our mechanism of the doublet-triplet Higgs mass splitting can work and the correct pattern of the GUT gauge symmetry breaking is realized. We propose the following superpotential as an example,

$$
W=\operatorname{tr}[(\bar{Q} Q) \bar{\Sigma}]+\operatorname{tr}\left[\Sigma^{2} \bar{\Sigma}\right]+Z\left(\operatorname{tr}\left[\Sigma^{2}\right]+\operatorname{tr}[\bar{Q} Q]-m^{2}\right)
$$

where $\bar{\Sigma}, \Sigma$ and $Z$ are superfields having charges $(\mathbf{1}, \mathbf{2 4}, 6 / 5),(\mathbf{1}, \mathbf{2 4}, 2 / 5)$ and $(\mathbf{1}, \mathbf{1}, 6 / 5)$, respectively, under $S U(5)_{H} \times S U(5)_{G U T} \times U(1)_{R}$, all the Yukawa coupling constants are taken to be 1 , for simplicity, and the explicit $U(1)_{R}$ symmetry breaking parameter $m$ is introduced. Note that, except the $m$ term, the above superpotential is general one consistent with all the symmetries. The first and the fourth terms lead to the mass terms for the effective field $M$ in the effective field description. The parameter $m$ is assumed to be the order of the GUT scale. Although this superpotential is simple, it should be regarded as the effective one because of the introduction of $m$. We give a comment on this point in the last section.

Considering the superpotentials of Eqs. (3) and (9), we find the SUSY vacuum such that $Z=0, \bar{\Sigma}=0, M=\operatorname{diag}(v, v, v, 0,0)$ and $\Sigma=\operatorname{diag}(2 \sigma, 2 \sigma, 2 \sigma,-3 \sigma,-3 \sigma)$ with $v=m^{2} / 9 \Lambda \sim$ $M_{G U T}$ and $\sigma= \pm m / 3 \sqrt{5} \sim M_{G U T}$. P Now, we obtain the desired SUSY vacuum where our mechanism of the doublet-triplet Higgs mass splitting can work, the GUT gauge symmetry is correctly broken to the standard model one, and all the light particle contents are the same as that of the MSSM.

Since, at low energy $\leq M_{G U T}$, the above model is described by the MSSM, the standard model gauge couplings are successfully unified at $M_{G U T} \sim 10^{16} \mathrm{GeV}$. At high energy larger than the GUT scale, the MSSM gives place to the $S U(5)_{G U T}$ GUT. Although the particle contents introduced above make the $S U(5)_{G U T}$ GUT asymptotic non-free, the gauge coupling remains in the perturbative regime below the Planck scale with appropriate large mass parameters, for example, $m_{1}, m_{2} \gg M_{G U T}$.

\footnotetext{
${ }^{5}$ There are other SUSY vacua where, for example, the triplet Higgs superfields are massless but the doublet Higgs superfields are heavy. We do not consider such a case since it is out of our interests.
} 


\section{SUMMARY AND COMMENTS}

We proposed a new mechanism towards the solution of the doublet-triplet Higgs mass splitting problem in the supersymmetric grand unified theory. Our model is based on the gauge group $S U(5)_{H} \times S U(5)_{G U T}$ with particle contents introduced in the sections II and III. All the fundamental superpotentials in our model are renormalizable and general, which are consistent with all the symmetries. In the present model, we obtained the desired SUSY vacuum, in which the doublet-triplet Higgs mass splitting is realized through the effect (the quantum deformation of moduli space) by the strong $S U(5)_{H}$ gauge dynamics, and the correct GUT gauge symmetry breaking takes place. At low energy, our model connects with the minimal supersymmetric standard model.

Here, we give some comments on more detailed features of our model. Let us first discuss the origin of the explicit $U(1)_{R}$ breaking term in Eq. (9). This term is necessary to realize the correct breaking pattern of the $S U(5)_{G U T}$ and to make the fields except the MSSM particle contents heavy. One possibility to provide this term is to introduce another strong gauge dynamics. For example, consider a sector based on the gauge group $S U(2)_{H}$ with four doublet superfields $Q_{i}(i=1,2,3,4)$ having the $U(1)_{R}$ charge $2 / 5$. In this case, the general renormalizable superpotential at the tree level is of the form

$$
W=-Z\left[Q_{i} Q_{j}\right]
$$

where [ ] denotes the contraction of the $S U(2)_{H}$ indices by the epsilon tensor. The strong $S U(2)_{H}$ gauge dynamics causes the quantum deformation of moduli space, and the constraint, $\operatorname{Pf}\left[Q_{i} Q_{j}\right]=\Lambda_{2}^{4}$, comes out [12]. Here, $\Lambda_{2}$ is the dynamical scale of the $S U(2)_{H}$ gauge interaction. Under this constraint, the above superpotential leads to the $U(1)_{R}$ symmetry breaking term $m=\Lambda_{2}$ in Eq. (9). This is the dynamical origin of the $S U(5)_{G U T} \times U(1)_{R}$ symmetry breaking through Eqs. (9) and (10). It is possible to construct other examples.

In this paper, we have considered only the SUSY vacuum. If the SUSY breaking effects are taken into account, the soft SUSY breaking terms in the scalar potential are induced, and the vacuum we discussed may be changed. Since our SUSY vacuum conditions are required at the GUT scale much larger than the typical SUSY breaking scale, the SUSY breaking effects are expected to be small. However, there is an interesting possibility that this small effects may generate the $\mu$ term for the doublet Higgs superfields. Indeed, analyzing the scalar potential based on the minimal supergravity scenario, we can find that nonzero $\mu$ term is really generated through the VEV of $M$. Unfortunately, it is too small,

$\mu \sim m_{3 / 2}^{2} / M_{G U T}$, as expected in the dimensional analysis, where $m_{3 / 2}$ is the electroweak scale gravitino mass. Therefore, we need another mechanism to provide the sizable $\mu$ term. For this issue, the Guidice-Masiero mechanism [17] is remarkable, since this mechanism automatically generates the $\mu$ term and the $B$ parameter with the electroweak scale through the SUSY breaking effects.

Finally, although the doublet-triplet Higgs mass splitting is simply realized through the strong gauge dynamics, our model is somewhat complicated. It may be possible to construct a simpler dynamical model with our mechanism. Also, in order to obtain a more complete model, we have to construct a concrete SUSY breaking sector, and combine it with the $S U(5)_{G U T}$ sector. These directions are worth investigating. 


\section{ACKNOWLEDGMENTS}

We would like to thank Yasuhiro Okada and Takeo Moroi for useful discussions. This work was supported by the JSPS Research Fellowships for Young Scientists (R.K.). 


\section{REFERENCES}

[1] See, for example, H.P Nilles, Phys. Rep. 110, 1 (1984).

[2] C. Giunti, C.W. Kim, and U.W. Lee, Mod. Phys. Lett. A 6 1745, (1991); U. Amaldi, W. de Boer, and H. Furstenau, Phys. Lett. B 260447 (1991); P. Langacker and M. Luo, Phys. Rev. D 44817 (1991).

[3] H. Georgi and S.L. Glashow, Phys. Rev. Lett. 32, 438 (1974).

[4] As recent works, see, for example, V. Lucas and S. Raby, Phys. Rev. D 55, 6986 (1997); T. Goto and T. Nihei, Phys. Rev. D 59, 115009 (1999).

[5] M.T. Grisaru, W. Siegel, and M. Rocek, Nucl. Phys. B 159, 429 (1997).

[6] E. Witten, Phys. Lett. 105B, 267 (1981); L. Ibanez and G.G. Ross, Phys. Lett. 110B, 215 (1982); D.V. Nanopoulos and K. Tamvakis, Phys. Lett. 113B, 151 (1982); S. Dimopoulos and H. Georgi, Phys. Lett. 117B, 287 (1982); D. Nemeschansky, Nucl. Phys. B 234, 379 (1984).

[7] H. Georgi, Phys. Lett. 108B, 283 (1982); A. Masiero, D.V. Nanopoulos, K. Tamvakis, and T. Yanagida, Phys. Lett. 115B, 380 (1982); B. Grinstein, Nucl. Phys. B 206, 387 (1982).

[8] T. Yanagida, Phys. Lett. B 344, 211 (1995); T. Hotta, K.-I. Izawa, and T. Yanagida, Phys. Rev. D 53, 3913 (1996); ibid D 54, 6970 (1996); K.-I. Izawa and T. Yanagida, Prog. Theor. Phys. 97, 913 (1997); Y. Imamura, T. Watari, and T. Yanagida, hep-ph/0103251.

[9] S. Dimopoulos and F. Wilczek, Report No. NSF-ITP-82-07 (1982); K.S. Babu and S.M. Barr, Phys. Rev. D 48, 5354 (1993); K.S. Babu and and R.N. Mohapatra, Phys. Rev. Lett. 74, 2418 (1995).

[10] K. Inoue, A. Kakuto, and H. Takano, Prog. Theor. Phys. 75, 664 (1986); A. Anselm and A. Johansen, Phys. Lett. B 200, 331 (1988); A. Anselm, Sov. Phys. JETP 67, 663 (1988); Z.G. Berezhiani and G. Dvali, Sov. Phys. Lebedev. Inst. Rep.5, 55 (1989) Z.G. Berezhiani, C. Csaki, and L. Randall, Nucl. Phys. B 44, 61 (1995).

[11] Y. Kawamura, Prog. Theor. Phys. 103, 613 (2000); G. Altarelli and F. Feruglio, hep-ph/0102301; A.B. Kobakhidze, hep-ph/0102323; L. Hall and Y. Nomura, hepph/0103125; M. Kakizaki and M. Yamaguchi, hep-ph/0104103.

[12] N. Seiberg, Phys. Lett. B 318, 469 (1993); N. Seiberg, Phys. Rev. D 496857 (1994); K. Intriligator, R.G. Leigh, and N. Seiberg, Phys. Rev. D 501092 (1994).

[13] J. Polchinski and L. Susskind, Phys. Rev. D 26, 3661 (1982); H.P. Nilles, M. Srednicki, and D. Wyler, Phys. Lett. B 122, 337 (1983).

[14] J. Bagger and E. Poppitz, Phys. Rev. Lett. 712380 (1993); J. Bagger, E. Poppitz, and L. Randall, Nucl. Phys. B 445, 59 (1995). H.P. Nilles and N. Polonsky, Phys. Lett. B 412, 69 (1997); C. Kolda, S. Pokorski, and N. Polonsky, Phys. Rev. Lett. 80, 5263 (1998).

[15] P. Ciafaloni and A. Pomarol, Phys. Lett. B 404, 83 (1997); Y. Chikira, N. Haba, and Y. Mimura, Phys. Rev. D 59, 055002 (1999).

[16] M. Dine and A.E. Nelson, Phys. Rev. D 48, 1277 (1993); M. Dine, A.E. Nelson, and Y. Shirman, Phys. Rev. D 51, 1362 (1995); M. Dine, A.E. Nelson, Y. Nir, and Y. Shirman, Phys. Rev. D 53, 2658 (1996).

[17] G. Giudice and A. Masiero, Phys. Lett. B 206, 1480 (1988). 